\title{
Density matrix embedding from broken symmetry lattice mean fields
}

\author{
Ireneusz W. Bulik, ${ }^{1}$ Gustavo E. Scuseria, ${ }^{1,2}$ and Jorge Dukelsky ${ }^{3}$ \\ ${ }^{1}$ Department of Chemistry, Rice University, Houston, Texas 77005, USA \\ ${ }^{2}$ Department of Physics and Astronomy, Rice University, Houston, Texas 77005, USA \\ ${ }^{3}$ Instituto de Estructura de la Materia-CSIC, Serrano 123, 28006 Madrid, Spain
}

(Received 30 September 2013; revised manuscript received 7 January 2014; published 27 January 2014)

\begin{abstract}
Several variants of the recently proposed density matrix embedding theory (DMET) [G. Knizia and G. K-L. Chan, Phys. Rev. Lett. 109, 186404 (2012)] are formulated and tested. We show that spin symmetry breaking of the lattice mean-field allows precise control of the lattice and fragment filling while providing very good agreement between predicted properties and exact results. We present a rigorous proof that at convergence this method is guaranteed to preserve lattice and fragment filling. Differences arising from fitting the fragment one-particle density matrix alone versus fitting fragment plus bath are scrutinized. We argue that it is important to restrict the density matrix fitting to solely the fragment. Furthermore, in the proposed broken symmetry formalism, it is possible to substantially simplify the embedding procedure without sacrificing its accuracy by resorting to density instead of density matrix fitting. This simplified density embedding theory (DET) greatly improves the convergence properties of the algorithm.
\end{abstract}

DOI: 10.1103/PhysRevB.89.035140

\section{INTRODUCTION}

Strongly correlated electron systems are currently of great interest in the condensed matter physics and quantum chemistry communities [1,2]. Despite the differences, for example, between frustrated lattices and chemical bond breaking, the strong-correlation phenomenon in these systems has similar roots which are manifested by the breakdown of the mean-field picture. Regardless of the details of the problem at hand, strong-correlation has significant impact on many important aspects of the physics of various systems and cannot be ignored [3-6]. A low computational cost, qualitatively correct description of strongly-correlated materials would have great impact on the quality of theoretical predictions. Hence the quest for developing novel, as well as improving existing approaches for strong-correlation continues unabated.

One of the reasons that robust methods for the treatment of strong correlation are so elusive is related to the size of the systems of current interest. With increasing number of electronic degrees of freedom, the numerical complexity of exactly solving the problem quickly becomes prohibitively large; approximate methods must be therefore employed. An ideal approximation should provide a systematic and qualitatively correct description that is computationally accessible and does not deteriorate with system size. Such an ideal tool would be therefore applicable to a broad range of problems. From a practical point of view, approximate methods should offer a good compromise between accuracy and computational cost. Many methods are available to tackle the strong-correlation problem; they include quantum and variational Monte Carlo [7-12], dynamical mean-field theory (DMFT) [13-22], density matrix renormalization group (DMRG) [23-26], methods based on symmetry breaking and restoration [27-32], and methods based on a Gutzwiller variational approach [33,34]. This list is not, by any means, exhaustive. The effort to develop new approximations is continuously undertaken.

Recently, Knizia and Chan introduced density matrix embedding theory (DMET), [35-37] a novel and promising tool as demonstrated by the high-quality results obtained
PACS number(s): 71.10.Fd, 71.27.+a, 71.30.+h

on Hubbard lattices. DMET has its roots in the embedding DMFT framework, where the complexity of the entire system is reduced by partitioning the problem into a fragment plus an entangled bath. Together, they constitute an impurity model. As opposed to DMFT, the DMET impurity model is frequencyindependent and therefore significantly simpler [35]. DMET is designed to reproduce the entanglement of the impurity rather than its Green's function. Moreover, the construction of the effective bath is achieved in an algebraic way. In particular, if the impurity Hamiltonian is defined in a basis derived from a product state, the complexity of the impurity basis construction amounts to a rather small matrix diagonalization [38]. Nonetheless, the extension of the DMET formalism to dynamic properties has recently been presented [37].

Density matrix embedding theory has been benchmarked for model 1D and 2D one-band Hubbard lattices [35] and in chemical systems [36]. In the present work, we focus on the former in order to further investigate the properties of this novel methodology. In particular, we analyze the convergence criterion employed in the initial study [35]. We follow the alternative criterion, introduced in Ref. [36] and prove that in periodic systems it allows us to gain full control over the lattice filling. Additionally, we investigate the impact of the effective bath basis on the quality of results. The formalism here presented allows for spin symmetry breaking in the underlying lattice mean-field solution that is used to construct the key ingredients of the DMET procedure [39]. The only symmetry constraint retained is lattice translational symmetry, although in the present work we understand it in an extended cell formalism. This means that the adopted translational unit cell is identical to the DMET fragment for which the calculations are performed. We also suggest an approximation that substantially reduces the number of parameters to be optimized. In particular, we show that it is sufficient to limit the density matrix fitting to diagonal elements, i.e., we propose, what we call density, as opposed to density matrix embedding. We provide a detailed numerical analysis of the results obtained with this approach. In particular, we benchmark the aforementioned approximation against high-quality reference 
data and exact solutions for energies, two-body correlation functions, and compressibility. The latter allows us to access the Mott gap in the Hubbard model [21].

\section{THEORY}

The present section is organized as follows. First, for clarity and to make this work self-contained, we review the basic principles of DMET. We discuss the properties of the embedding basis and sketch the algorithm. We then proceed to discuss the convergence criterion, which is by no means unique. The suggested route to incorporate broken symmetry embedding is described subsequently.

\section{A. Density matrix embedding theory}

In order to outline the key ideas of DMET, let us assume that we are given the exact ground state $|\Psi\rangle$ for the system of interest. Then, it is possible to perform a Schmidt decomposition of this wave function according to Ref. [40]:

$$
|\Psi\rangle=\sum_{i} \lambda_{i}\left|\alpha_{i}\right\rangle\left|\beta_{i}\right\rangle,
$$

where $\left|\alpha_{i}\right\rangle$ and $\left|\beta_{i}\right\rangle$ can be chosen such that the former represents a particular set of lattice site states, which we call the fragment. The latter must then be the complement that spans all the sites excluded from $\left|\alpha_{i}\right\rangle$, and we refer to it as the bath. The summation in the above equation is limited by the dimension of the smaller of these two sets. These bases are then used to project the Hamiltonian into the Schmidt states of the fragment $\left|\alpha_{i}\right\rangle$ and bath $\left|\beta_{i}\right\rangle$ [35]:

$$
\hat{H} \rightarrow \sum_{i j k l}\left|\alpha_{i}\right\rangle\left|\beta_{j}\right\rangle\left\langle\alpha_{i}\left|\left\langle\beta_{j}|\hat{H}| \alpha_{k}\right\rangle\right| \beta_{l}\right\rangle\left\langle\alpha_{k}\right|\left\langle\beta_{l}\right|=\hat{H}_{\mathrm{imp}} .
$$

Details of matrix elements of the projected Hamiltonian, usually refer to as impurity Hamiltonian, are not relevant here. For a discussion regarding the impurity Hamiltonian, the reader is referred to Ref. [35]. The key point to notice is that the size of the new basis (though many-body in principle) can be chosen much smaller than that of the original problem, if formulated in a single-particle basis. As discussed in Ref. [35], the original Hamiltonian $\hat{H}$ and the impurity Hamiltonian $\hat{H}_{\text {imp }}$ share the same ground state $|\Psi\rangle$; information about the expectation values of $\hat{H}$ can be extracted by studying $\hat{H}_{\text {imp }}$.

Unfortunately, in practical applications, we do not have access to the exact solution. The embedding states have to be approximated. The fundamental simplification of DMET is to replace the exact solution with a mean-field (here understood as Hartree-Fock) wave function, which is a simple product wave function ansatz. In this case, the Schmidt fragment and bath bases can be represented in terms of single-particle states. This greatly simplifies the computational treatment of the impurity problem and, as we show in this section, provides an effective truncation of the dimension of single-particle basis for the problem at hand.

Let us follow the procedure more closely on a specific example, a Hubbard Hamiltonian. In this case,

$$
\hat{H}=\sum_{i j, \sigma}\left(t_{i j}+v_{i j}\right) c_{i \sigma}^{\dagger} c_{j \sigma}+U \sum_{i} c_{i \uparrow}^{\dagger} c_{i \uparrow} c_{i \downarrow}^{\dagger} c_{i \downarrow},
$$

where $t_{i j}$ connects nearest neighbors, $U$ is the on-site repulsion parameter, and $v$ is an effective one-body potential that we wish to introduce; the rationale behind this potential will become apparent in the following. At this point, let us just notice that $v$ is contained within the fragment and it is periodically replicated over the lattice. In the limit of vanishing potential $v$, the Hamiltonian reduces to the standard Hubbard model.

The first step in DMET is to solve the above problem at the mean-field level. This procedure yields a single Slater determinant $|\Phi\rangle=\Pi_{p} a_{p}^{\dagger}|0\rangle$, where $a^{\dagger}$ creates a hole (occupied) state $|\phi\rangle$ and $|0\rangle$ is a bare vacuum. The product runs from 1 to the number of electrons. The hole creation operators are defined by the underlying Hartree-Fock transformation $\mathbb{D}$ from physical fermions $c^{\dagger}$ [41],

$$
a_{p}^{\dagger}=\sum_{\mu} \mathbb{D}_{\mu p} c_{\mu}^{\dagger} .
$$

The basis $\mu$ takes into account the position and the spin degrees of freedom. In order to construct the fragment and bath states needed to define the impurity Hamiltonian, we introduce the operator $\hat{P}_{F}$ that projects the hole levels onto the single-particle basis contained within the fragment. Additionally, we have the complement $\hat{P}_{B}$ such that $\hat{P}_{F}+\hat{P}_{B}=\hat{I}$, where $\hat{I}$ is the identity operator. Following Ref. [38], one can define an overlap matrix $\mathbb{M}$,

$$
\mathbb{M}_{p q}=\left\langle\phi_{q}\left|\hat{P}_{F}\right| \phi_{p}\right\rangle,
$$

where the indices $p$ and $q$ run over hole states. This Hermitian matrix can be brought to diagonal form by a unitary transformation $\mathbb{V}$ satisfying $\mathbb{V}^{\dagger} \mathbb{M} \mathbb{V}=d$, where $d$ contains at $\operatorname{most} \min \left(n_{e}, n_{f}\right)$ nonzero eigenvalues (here, $n_{e}$ is the number of electrons in the lattice whereas $n_{f}$ is the size of the single-particle fragment basis). In the following, we will assume that $n_{e} \geqslant n_{f}$ and that all $n_{f}$ eigenvalues are different from 1 and 0 (otherwise, special care has to be taken while constructing the fragment and bath states). For each of the nonzero eigenvalues, one may construct a fragment state,

$$
\left|f_{i}\right\rangle=\sum_{p} \frac{\mathbb{V}_{p i}^{*}}{\sqrt{d_{i}}} \hat{P}_{F}\left|\phi_{p}\right\rangle
$$

and a bath state,

$$
\left|b_{i}\right\rangle=\sum_{p} \frac{\mathbb{V}_{p i}^{*}}{\sqrt{1-d_{i}}} \hat{P}_{B}\left|\phi_{p}\right\rangle
$$

The single-particle states that correspond to vanishing eigenvalues of $\mathbb{M}$ are considered as the inert core states $|i\rangle$. Following Ref. [40], the states corresponding to vanishing eigenvalues of $\mathbb{M}$ denote orbitals with zero probability of being in the fragment space. Similarly, one can think of the inert core states as states with vanishing coupling to the fragment states in the mean-field one-particle density matrix. The reader is referred to Appendix A 2 for more details.

In DMET, the inert core single-particle states are eliminated from the impurity problem (i.e., they are not included while projecting the Hamiltonian onto the impurity Hamiltonian). Therefore DMET retains only a small portion of the large number of single-particle states constituting the original Hilbert space of the problem. In fact, the total dimension of 
the single-particle basis is just twice the dimension of the single-particle basis spanned by the fragment. This is clear from the particular properties of the spectrum of $\mathbb{M}$ [Eq. (5)] for the mean-field wave function. In other words, the Hilbert space of the impurity model is significantly smaller than that of the original problem. It is also determined by the size of the fragment.

Armed with fragment and bath single-particle states, which we shall refer to as an embedding basis, one can construct an impurity Hamiltonian (henceforth, for brevity of notation, the indices in the impurity Hamiltonian denote spin and space coordinates),

$$
\hat{H}_{\mathrm{imp}}=\sum_{i j} \tilde{t}_{i j} d_{i}^{\dagger} d_{j}+\frac{1}{4} \sum_{i j k l} \tilde{U}_{i j k l} d_{i}^{\dagger} d_{j}^{\dagger} d_{l} d_{k}+\sum_{i j} \tilde{v}_{i j} b_{i}^{\dagger} b_{j} .
$$

In the above, $b^{\dagger}$ denotes bath creation operators whereas $d^{\dagger}$ denotes either fragment or bath ones. The $\tilde{t}$ and $\tilde{U}$ denote the one- and (antisymmetrized) two-body terms of the Hubbard Hamiltonian projected onto the embedding basis, respectively. Similarly, $\tilde{v}$ corresponds to the additional effective potential. At this point, we would like to point out that this potential, introduced in Eq. (3), does not affect directly the one-body part of the impurity Hamiltonian in the fragment space. In other words, the fragment part of the impurity Hamiltonian corresponds to the physical Hamiltonian. As the reader may notice, the DMET impurity Hamiltonian derived from the Schmidt decomposition of the mean-field wave function is expressed in terms of single-particle states and corresponding creation and annihilation operators of fragment and bath states. This is to be compared with Eq. (2), which is more general and may include many-body states as a basis.

The impurity Hamiltonian is the central part of the approximation. As the dimension of the embedding basis is significantly reduced (by means of eliminating the inert core $|i\rangle$ states), one is now in a position to employ powerful ground state computational schemes for solving it. In particular, exact diagonalization becomes computationally feasible for modest fragment sizes.

The goal of DMET is to find an optimal effective one-body potential $v$ by means of minimizing

$$
\left|\gamma-\gamma_{0}\right|=\left|\left\langle\Psi_{\text {imp }}\left|d^{\dagger} d\right| \Psi_{\text {imp }}\right\rangle-\left\langle\Phi\left|d^{\dagger} d\right| \Phi\right\rangle\right| .
$$

In the above, $\gamma$ and $\gamma_{0}$ are the one-particle density matrix evaluated with the impurity and the mean-field wave function, respectively. The precise meaning of the convergence criterion is discussed in the following section.

Once converged, the energy density $e$ for the fragment can be evaluated as

$$
e=\sum_{f j} \tilde{t}_{f j} \gamma_{j f}+\frac{1}{4} \sum_{f j k l} \tilde{U}_{f j k l} \Gamma_{k l f j},
$$

where $\Gamma_{k l f j}=\left\langle d_{f}^{\dagger} d_{j}^{\dagger} d_{l} d_{k}\right\rangle$ is the two-particle density matrix. The index $f$ in the above summations implies that at least one of the basis functions has to belong to the fragment space. Clearly, the energy expression does not correspond to the expectation value taken with respect to the full lattice Hamiltonian. Because the above expression is not a true expectation value taken with respect to the full Hamiltonian of the system, the DMET energy need not be an upper bound to the exact value. In practice, we have observed a tendency of the procedure to deliver ground-state energies that are below the exact ones.

The self-consistency loop in DMET takes the following form: (1) obtain an initial guess for $v$ [Eq. (3)], (2) find $\mathbb{D}$ of Eq. (4) and construct fragment and bath states according to Eqs. (5)-(7), (3) construct the impurity Hamiltonian (8) and solve it, (4) update $v$ by means of Eq. (9), and (5) if the update is not negligible, replicate the fragment potential $v$ over the entire lattice and go to step 2.

\section{B. Convergence criterion}

DMET provides a very good compromise between accuracy and computational cost for the Hubbard Hamiltonian [35]. However, in the original formulation, the authors chose to define a convergence criterion based on an effective one-body potential that minimizes the difference between the correlated and mean-field one-particle density matrices over the full impurity space, i.e., fragment and bath basis. In other words, the effective potential sought satisfies

$$
\min _{v} \sum_{i j}\left|\gamma-\gamma_{0}\right|_{i j}
$$

where the indices $i$ and $j$ run over the fragment and the bath states. This approach, despite its merits, introduces a certain limitation to the model. Primarily, as shown in Appendix A 2, because the mean-field density matrix in the embedding basis must be idempotent, it is not possible to find $v$ such that the fit between the mean-field $\left(\gamma_{0}\right)$ and correlated one-particle density matrix $(\gamma)$ is exact. An accurate impurity solver yields $\gamma$ with eigenvalues different from 1 and 0 , except for special cases, like, for example, a trivial system of noninteracting particles. One therefore concludes that in general $\sum_{i j}\left|\gamma-\gamma_{0}\right|_{i j} \neq 0$.

This deficiency is not just a formal issue. In fact, since the match between the density matrices cannot be perfect, the average number of particles that the impurity Hamiltonian treats cannot be controlled and can deviate from the desired value. As clear from Eq. (10), the total energies for the physical system computed with DMET are determined by the fragment energy. It follows then, that any error in the average number of particles in the fragment affects the predictions for the entire system. This problem can be ameliorated by changing the definition of the converged effective one-body potential. An alternative convergence criterion can be therefore formulated [36],

$$
\min _{v} \sum_{i j \in f}\left|\gamma-\gamma_{0}\right|_{i j}
$$

Henceforth, the block of the density matrix in the embedding basis with two indices located on the fragment is referred to as fragment one-particle density matrix. With the above definition of the effective potential, provided that the minimum corresponds to a perfect match, the average number of electrons per fragment is correct. This statement is particularly important from the viewpoint of periodic systems, where the average particle density per fragment is known. The proof of this statement is presented in Appendix A 4. 
The above considerations narrow the choices for the convergence criterion due to the constraint on the number of particles per fragment. Nonetheless, fitting only the fragment one-particle density matrix is not a unique choice. For this reason, our density embedding proposal further simplifies the numerical procedure while imposing control over the lattice filling. As shown in Appendix A 4, an exact match between density matrices guarantees that the trace of fragment $\gamma$ will have the desired number of particles. Since the trace is obviously determined by the diagonal elements of the onebody density matrix, we formulate the convergence criterion as

$$
\min _{v} \sum_{i \in f}\left|\gamma-\gamma_{0}\right|_{i i}
$$

The decrease in the number of parameters that needs to be optimized is accompanied by simplifications of the effective lattice Hamiltonian that one should solve,

$$
\hat{H}=\sum_{i j, \sigma}\left(t_{i j}+v_{i j} \delta_{i j}\right) c_{i \sigma}^{\dagger} c_{j \sigma}+U \sum_{i} c_{i \uparrow}^{\dagger} c_{i \uparrow} c_{i \downarrow}^{\dagger} c_{i \downarrow} .
$$

We find that Eq. (13) greatly facilitates convergence while delivering results quantitatively similar to those obtained with the full method. For the purpose of the present work, we shall denote the results obtained with the convergence criterion defined by Eq. (13) as DET. In this approach, the effective potential has a clear physical meaning as an effective (site dependent) chemical potential.

\section{Spin symmetry broken formalism}

Having discussed the convergence criterion in DMET, let us turn our attention to possibilities for optimizing the embedding basis. The initial DMET calculations neglected the effect of two-body interactions in the lattice [35]. In other words, the two-body interactions were suppressed both in the lattice Hamiltonian and bath portions of the impurity Hamiltonian. Only particles in the fragment states were subjected to the on-site repulsion. Since in mean field the entire system is approximated by a set of noninteracting particles, the Hartree-Fock transformation constitutes the exact solution; no symmetry breaking can occur, with some exceptions (here, we do not consider explicit symmetry breaking via an effective potential [42]). For example, due to lattice discretization into a set of fragments, translational symmetry might be violated; this, however, would again correspond to a situation where one works in an extended unit cell framework. Another possibility is degeneracy of the solution where the wave function may be chosen to violate certain symmetries of the Hamiltonian.

In the present work, we adopt a different procedure. The full lattice Hamiltonian corresponds exactly, apart from the effective one-body potential whose role has been already discussed, to the Hubbard Hamiltonian. The system is then treated with a spin-unrestricted formalism, where the Hartree-Fock wave function need not be an eigenfunction of the $\hat{S}^{2}$ operator. This procedure leads to a spin-dependent embedding basis and an impurity Hamiltonian that does not need to commute with the $\hat{S}^{2}$ operator. In order to retain the simplicity of a spin-restricted formulation, we do not choose the effective one-body potential to be spin-dependent. As a direct consequence, we define the effective one-body potential by

$$
\min _{v}\left|\gamma^{c}-\gamma_{0}^{c}\right| \text {. }
$$

Here, $\gamma_{c}=1 / 2\left(\gamma_{\uparrow \uparrow}+\gamma_{\downarrow \downarrow}\right)$ is the charge density. The fitted one-particle density matrix may be chosen either as DMET or DET type. On the other hand, we note that the broken spin symmetry formalism does not easily support fitting of the entire one-particle density matrix in the embedding basis as was done in Ref. [35]. Let us first stress that the transformation of the bare fermion basis to the embedding basis is a projection and hence cannot be inverted (unless, of course, one chooses to divide the whole system into two equal fragments). Only the transformation of the fragment states is unitary (and hence invertible). Therefore solely the $\uparrow$ and $\downarrow$ fragment density matrices are expressible in the common basis that defines a charge density matrix. For this reason, fitting of the entire one-particle density matrix has no clear physical meaning, though it may be numerically performed. We stress that the arguments outlined above regarding correct filling are directly applicable to the charge density matrix. Indeed, in a spinrestricted formalism, the charge density matrix and the oneparticle density matrix are equivalent.

Finally, let us note that away from half-filling, the spin unrestricted mean-field solution can admit charge fluctuations that are beyond the size of the fragment. In order to maintain the mean-field calculations commensurate with the chosen fragment size, we solve the Hartree-Fock equations in momentum space assuming homogeneity of the fragment superlattice.

\section{RESULTS AND DISCUSSION}

In the following sections, we benchmark all the embedding schemes discussed above for the 1D Hubbard model. For clarity, let us define acronyms that will be used in the rest of this paper. Results labeled as BA correspond to the exact Bethe ansatz solution [43]. Calculations denoted as DMET(n) refer to calculations where spin symmetry $\left(\hat{S}^{2}\right)$ in the mean-field solution is allowed to break and fitting of the one-particle density matrix is performed over the entire fragment chosen to include $n$ sites. Similarly, DET(n) denotes calculations where the fit is enforced only on the diagonal elements of the fragment one-particle density matrix. In order to better illustrate the performance of the broken symmetry approach, we also include data obtained with embeddings where the two-body interaction is suppressed in the lattice. To be more precise, in these schemes, the Hamiltonian for which the mean-field solution is obtained corresponds to Eq. (3) with $U=0$, however, the impurity Hamiltonian does include the on-site interaction but only in the fragment space. These, as already discussed, correspond to a noninteracting case and are denoted as $\mathrm{NI}$ and $\mathrm{NI}_{F}$. The additional subscript $F$ (for "Full" matrix) implies fitting of the full impurity one-particle density matrix (this is the method introduced in Ref. [35]). For convenience, Table I includes the key qualities of all studied embedding schemes.

\section{A. Half-lattice embedding}

We now focus on a particular benchmark case where the entire lattice consists of only two fragments. In such a system, 
TABLE I. Comparison of the key qualities of all studied embedding schemes. Equations (11)-(13) correspond to fitting of full impurity, fragment only, and the diagonal of the fragment one-particle density matrix, respectively. Two-body denotes inclusion of these terms in the lattice and the bath portions of the impurity. Spin symmetry denotes whether the mean-field solution is required to be an eigenstate of the $\hat{S}^{2}$ operator.

\begin{tabular}{lcccc}
\hline \hline Method & Fitting & Spin symmetry & Two-body & Reference \\
\hline DMET & Eq. (12) & NO & YES & This work \\
DET & Eq. (13) & NO & YES & This work \\
NI & Eq. (12) & YES & NO & This work \\
NI $_{F}$ & Eq. (11) & YES & NO & Ref. [35] \\
\hline
\end{tabular}

at half-filling, the Schmidt decomposition is just a unitary transformation of the bare fermion basis. The inert core states are absent. Therefore the complexity of solving the impurity Hamiltonian is equivalent to solving the original problem. Results in Table II confirms that the DMET embedding scheme is not exact in this case. This is because the exact and mean-field one-particle density matrices are not the same. In particular, they differ in the fragment space. The DMET equations are not immediately satisfied and the effective potential has to be optimized. This leads to a slight but significant deviation of total energies from the exact ones. The situation is different for the DET scheme. The mean-field solution charge density does not break translational symmetry and carries proper filling. This is the case for the exact answer as well. For this reason, the diagonal elements of both matrices agree. The optimal effective potential vanishes and the impurity Hamiltonian coincides with the Hubbard model. The DET embedding scheme converges in one iteration and the computed energy is equal to the exact one.

\section{B. Hubbard rings at half-filling}

In order to assess the performance of DMET and DET formalisms, we study the 1D Hubbard model at half-filling. In the present section, the calculations are performed for a ring of 400 sites with periodic boundary conditions. The exact solution for half-filling was obtained at the thermodynamic

TABLE II. Energy per site (in units of $t$ ) for small Hubbard rings at half-filling evaluated with DMET and DET when the entire lattice is divided into two identical fragments. Exact values are shown for comparison.

\begin{tabular}{lccccc}
\hline \hline & \multicolumn{5}{c}{8 sites } \\
$U=$ & 2 & 4 & 6 & 8 & 10 \\
Exact & -0.8210 & -0.5754 & -0.4261 & -0.3333 & -0.2721 \\
DMET(4) & -0.8382 & -0.5609 & -0.4118 & -0.3210 & -0.2616 \\
DET(4) & -0.8210 & -0.5754 & -0.4261 & -0.3333 & -0.2721 \\
\hline \multicolumn{5}{c}{4 sites } \\
$U=$ & 2 & 4 & 6 & 8 & 10 \\
Exact & -0.7071 & -0.5257 & -0.4087 & -0.3301 & -0.2750 \\
DMET(2) & -0.7262 & -0.5090 & -0.3860 & -0.3091 & -0.2568 \\
DET(2) & -0.7071 & -0.5257 & -0.4087 & -0.3301 & -0.2750 \\
\hline \hline
\end{tabular}
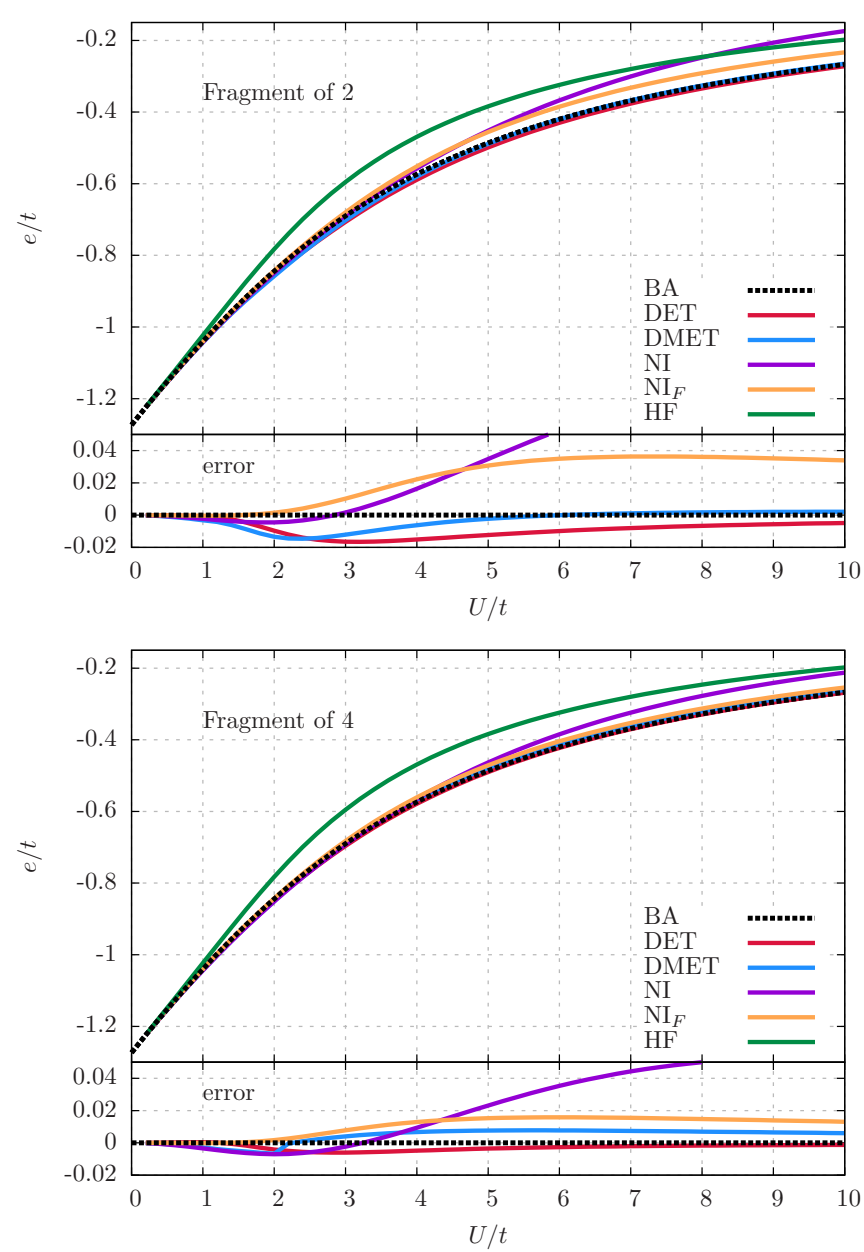

FIG. 1. (Color online) Energy per site of the 1D Hubbard model at half-filling evaluated with various embedding approximations with a fragment of two (top) and four sites (bottom). Bethe ansatz (BA) and Hartree-Fock (HF) results are added for comparison. The error with respect to BA is plotted in the bottom panel. The HF wave function is not constrained to preserve $\hat{S}^{2}$ symmetry.

limit [43-46]. Errors arising from finite size effects are negligible for a ring of 400 sites.

In Fig. 1, we compare calculations performed with DMET, DET, NI, and $\mathrm{NI}_{F}$. The calculations for $\mathrm{NI}_{F}$ were performed using the program published by Knizia and Chan [35] (we have verified that differences in boundary conditions are negligible by performing the NI calculations using our current implementation and the one in Ref. [35]).

For a small fragment composed of just two sites, we note that the spin unrestricted embedding scheme provides already a very good description of the energy for a broad range of on-site interaction strengths. The difference between DMET and DET is insignificant within the energy scale of the figure. This observation supports our choice of determining the optimal fit between the mean-field and correlated one-particle density matrices. The performance of embedding methods based on noninteracting lattice electrons is somewhat worse, although they still provide an accurate description, especially for smaller $U$ values. The presented data indicates that $\mathrm{NI}_{F}$ is superior to NI. Similar results are obtained with a fragment size of 
four sites. For this bigger fragment, we observe a systematic improvement of all the embedding schemes. In particular, broken spin DET and DMET are virtually indistinguishable from the exact answer within the scale of the figure. The $\mathrm{NI}_{F}$ data closely follow the BA curve as well. Finally, we notice that all the embedding schemes presented (apart from NI with large value of $U$ ) constitute a significant improvement with respect to the Hartree-Fock energy. As shown in Fig. 1, Hartree-Fock, even without constraints to preserve $\hat{S}^{2}$ symmetry, deviates significantly from BA, except for $U \rightarrow 0$ and $U \rightarrow \infty$ limits.

In order to gain further insight into the 1D Hubbard halffilled case, we investigate the two-body correlation function $\left\langle n_{\uparrow} n_{\downarrow}\right\rangle$ (on-site double occupancy). Indeed, a proper description of energetics combined with accurate double occupancy expectation values implies that the individual components (one- and two-body contributions) must be qualitatively correct. The correlation functions for the various embedding schemes are computed according to

$$
\left\langle n_{\uparrow} n_{\downarrow}\right\rangle=\frac{\langle\hat{U}\rangle}{U n_{f}},
$$

where $\hat{U}$ is the two-body interaction operator in the fragment (computed with the impurity wave function) and $n_{f}$ is the fragment size. For the exact solution, the double occupancy is computed using the Hellmann-Feynman theorem [45] as

$$
\left\langle n_{\uparrow} n_{\downarrow}\right\rangle=\frac{\partial e}{\partial U} .
$$

Results are presented in Fig. 2. Once more, the broken symmetry formalism, even with the smallest fragment, is highly accurate in the whole range of $U$ values investigated. Just as in the case of the energy, fitting of the entire fragment density matrix versus its diagonal provides very similar $\left\langle n_{\uparrow} n_{\downarrow}\right\rangle$. This implies that DMET and DET yield not only similar total energies but also individual components. The embedding schemes that neglect two-body interactions at the mean-field level give rise to double occupancy that departs significantly from exact results. In particular, NI highly overestimates this correlation function. This in turn translates into a notably high energy, especially in the strong coupling regime. On the other hand, $\mathrm{NI}_{F}$ yields improved behavior of $\left\langle n_{\uparrow} n_{\downarrow}\right\rangle$ as a function of $U$ in the strong coupling regime. However, the shape of the curve for intermediate couplings reveals some discrepancies as compared to BA. Increasing the size of the fragment, one notices an improvement in the trends of double occupancy as a function of $U$. DMET and DET results are virtually indistinguishable form the exact answer over the whole studied region. Not much worse are the $\mathrm{NI}_{F}$ results. Only in the case of NI, the overestimation of double occupancy in the strong coupling regime is noticeable. This agrees well with the underestimation of the correlation energy of the NI approach, which is not ameliorated by increasing the size of the embedded fragment.

As clear from the presented data, the embedding schemes included in the present study are exact in the noninteracting limit $(U=0)$. Furthermore, one could clearly notice that DMET, DET, and $\mathrm{NI}_{F}$ errors in the energy density and double occupancy tend to zero with increasing value of $U$ (i.e., towards the atomic limit). This is due to the effective
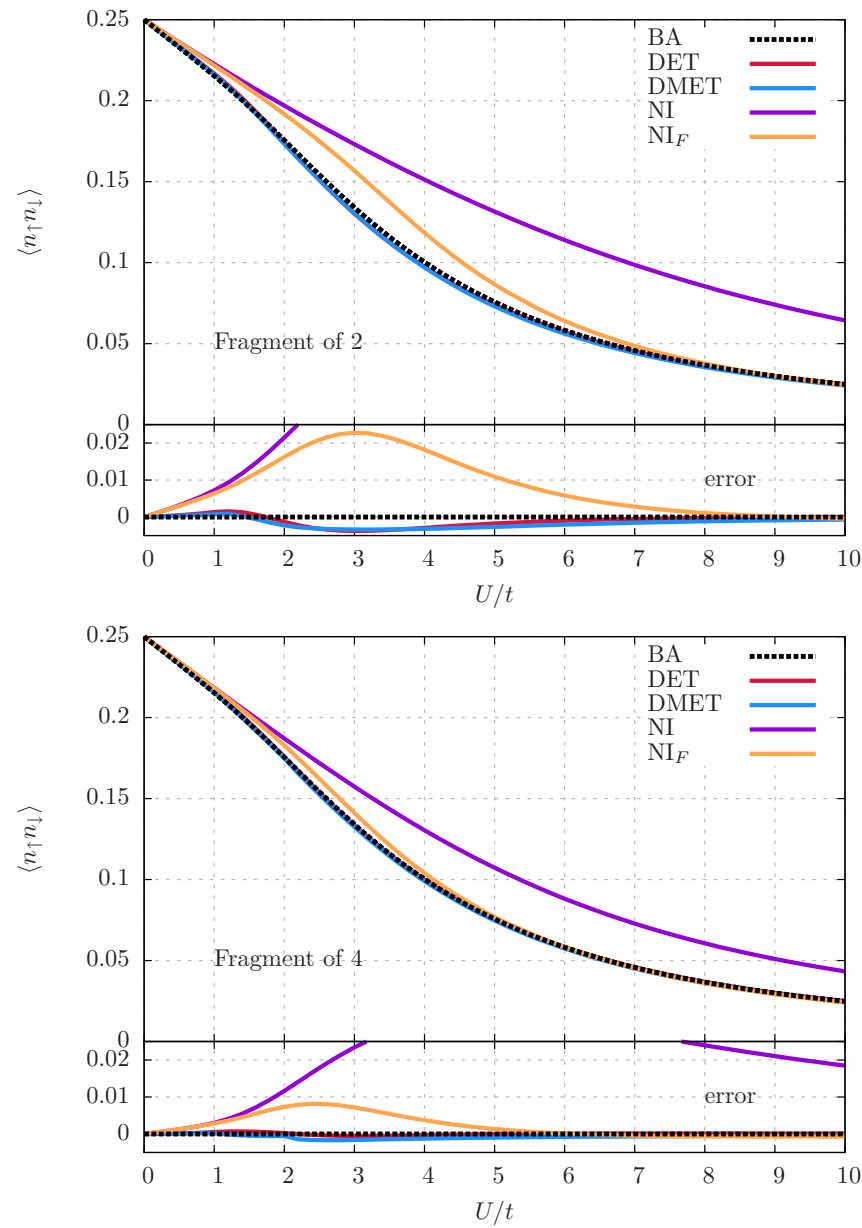

FIG. 2. (Color online) Comparison of double occupancy for the 1D Hubbard model at half-filling evaluated with various embedding approximations with a fragment of two (top) and four sites (bottom). Bethe ansatz (BA) results are added for comparison. The error with respect to BA is plotted in the bottom panel.

decoupling of the fragment and bath, which is exact in the atomic limit, as discussed in Ref. [35].

Finally, let us compare the ground state energy density of DET and DMET with recent variational cluster approach (VCA) [47] and cellular dynamical mean-field theory (CDMFT) [48] calculations. For the on-site interaction $U=4 t$ and $8 t$, the maximum relative error with respect to Bethe anstatz for the two sites fragment DET or DMET is $2.6 \%$ and $2.0 \%$, respectively, whereas for four sites fragment DET or DMET $1.2 \%$ and $2.1 \%$. In comparison, VCA calculations for $U=4 t$ become more accurate than $\mathrm{D}(\mathrm{M}) \mathrm{ET}(2)$ for a cluster size of six sites, and achieve similar accuracy to D(M)ET(4) with a cluster size of ten. For $U=8 t, \mathrm{D}(\mathrm{M}) \mathrm{ET}$ is as accurate as the VCA approach with a cluster of ten sites. The accuracy of $\mathrm{D}(\mathrm{M}) \mathrm{ET}$ is also comparable with CDMFT with similar cluster sizes.

\section{Hole-doped Hubbard rings}

In this section, we investigate the behavior of the symmetry broken embedding formalism in the hole-doped Hubbard 


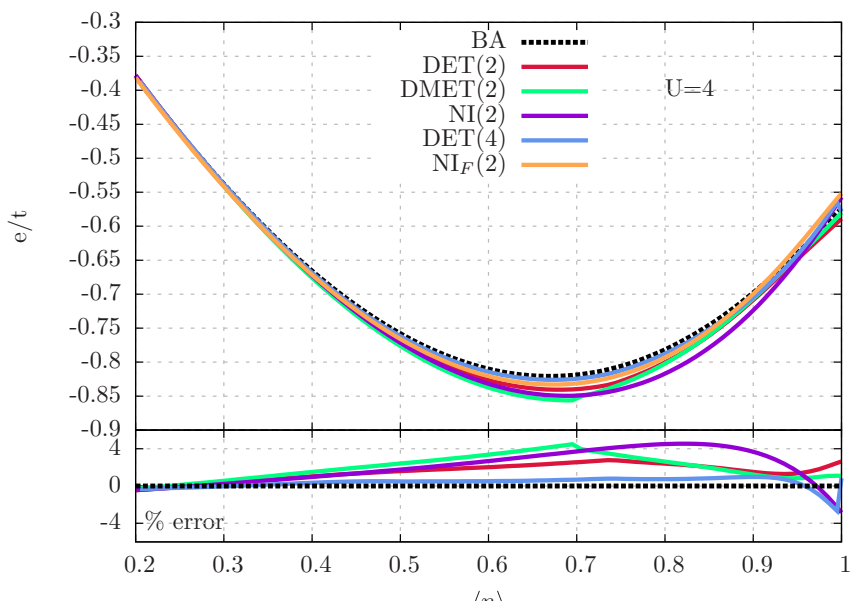

$\langle n\rangle$

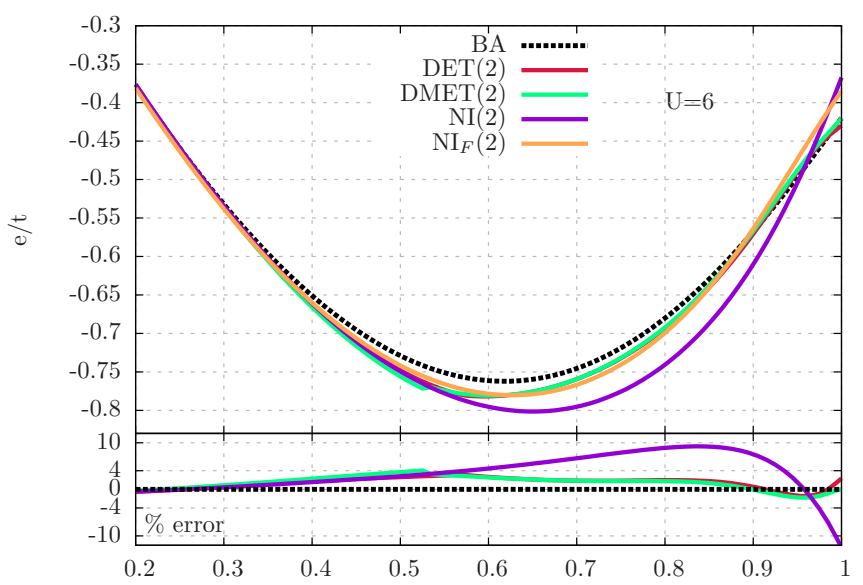

$\langle n\rangle$

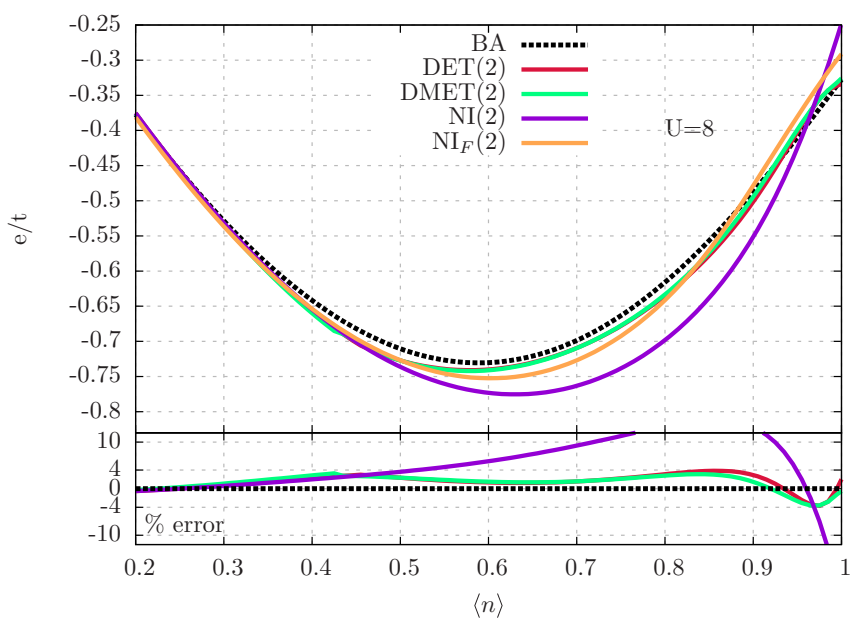

FIG. 3. (Color online) Energy density of the 1D Hubbard model as a function of hole doping evaluated with various embedding approximations for $U=4 t$ (top), $6 t$ (middle), and $8 t$ (bottom). Bethe ansatz (BA) results are added for comparison. The error with respect to $\mathrm{BA}$ is plotted in the bottom panel.

lattice. Again, the calculations correspond to a lattice composed of 400 sites.

In Fig. 3, we present the dependence of the energy per site as a function of lattice filling for three values of the on-site interaction, $U=4 t, 6 t$, and $8 t$. Additionally, we plot the relative error in the total energy as compared to exact values. The errors for $\mathrm{NI}_{F}$ are not included because the data was generated using the program of Ref. [35] where different periodic boundary conditions were used. The calculations are performed with a fragment of two, except for the $U=4 t$ curve evaluated with DET and a fragment of four. We do not present a more detailed investigation with larger fragments because of convergence problems with DMET. Indeed, we find that inclusion of twobody interactions in the lattice Hamiltonian introduces significant problems in the potential optimization; these problems, whatever their origin, do not appear in the DET density embedding scheme. For instance, even at $U=4 t$, we did not succeed in converging DMET equations for the entire $e(\langle n\rangle)$ curve.

As it is apparent from Fig. 3, all embedding schemes provide a fairly accurate description of energetics with the exception of NI. This embedding scheme quickly breaks down in the strong-coupling limit. Whereas for $U=4 t$ the computed energy does not bear an error of more than around 4\%, the situation drastically changes for larger on-site interaction strengths. In particular, the relative error compared to the exact answer easily goes above $10 \%$. Such a large error is in agreement with the discussion in the previous section for the half-filed case. The other embedding schemes, $\mathrm{NI}_{F}$, DMET, and DET significantly ameliorate this deficiency. For all values of $U$ tested, DET does not deviate from the BA by more than a few percent. Comparing broken symmetry methods to $\mathrm{NI}_{F}$, we notice that for $U=4 t$, the latter seems to perform somewhat better, especially for large doping fractions. Nonetheless, results obtained with the symmetry broken formalism compares favorably with the BA results.

For larger values of $U$, DMET and DET become more accurate. Their difference is mostly pronounced for lattice fillings above 0.9. Indeed, for relatively small doping fractions and a fragment of two sites, the broken symmetry formalism seems to provide a very accurate description. Finally, let us note that for all values of $U$ studied, broken symmetry DMET and DET calculations yield very similar results. Nevertheless, we would like to stress that DET introduces significant simplifications to the DMET numerical procedure. These simplifications translate directly into superior convergence for the nonlinear equations defining the embedding procedure.

Because of the large slope of the $e(\langle n\rangle)$ curve, we would like to stress that the present DMET and DET schemes have full control over the lattice filling. No error does therefore arise from deviations between electron number per fragment compared to lattice filling.

Increasing the size of the embedded fragment, we observe improvement with respect to two sites for $U=4 t$. The correction is most visible for larger doping fractions where the calculations with smaller fragments yield somewhat overcorrelated energies. Nonetheless, there is still room for improvement, especially at small doping fractions. This is the regime where DET(4) differs from the exact answer by around $3 \%$. For lattice filling below 0.95 , the relative error drops and the calculations give answers with errors within $1 \%$. Finally, let us point out that due to the fact that after a certain doping fraction, the mean-field no longer breaks spin symmetry, there is a slight discontinuity in the DMET and DET curves. 


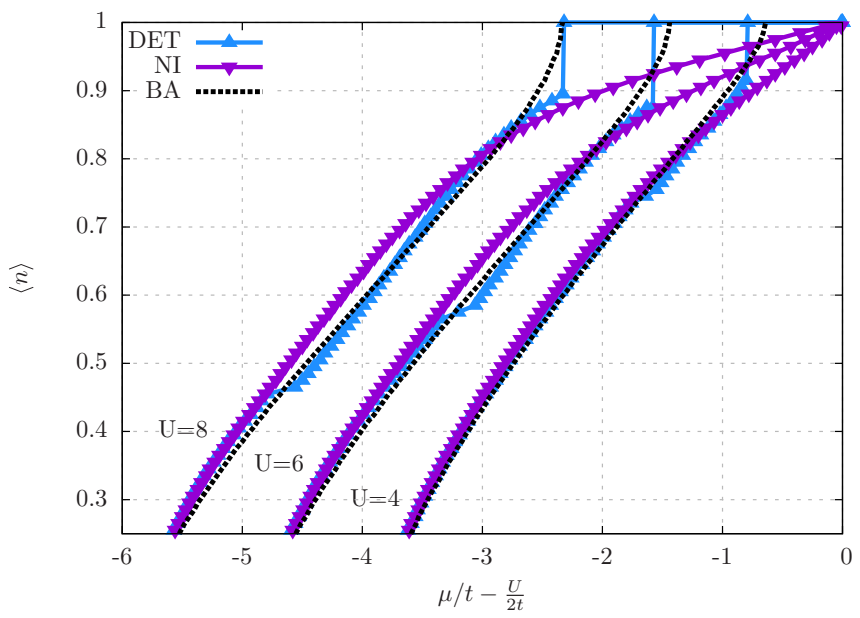

FIG. 4. (Color online) Lattice filling as a function of chemical potential evaluated with various embedding approximations. The embedded fragment consists of two sites in each case. Exact Bethe ansatz (BA) values are shown for comparison.

Let us now proceed to investigate the lattice density as a function of chemical potential. Results are presented in Figs. 4 and 5 and are obtained by minimizing

$$
\hat{H}=\hat{H}_{0}-\mu \hat{N}
$$

with respect to electron number at a given chemical potential $\mu$. Here, $\hat{H}_{0}$ corresponds to the Hubbard Hamiltonian and $\hat{N}$ is the number operator. In this work, we compare data obtained with the symmetry broken embedding formalism against the NI one. Analogous results obtained with $\mathrm{NI}_{F}$ can be found in Ref. [35]. See also Ref. [49].

Whereas the $\mathrm{NI}_{F}$ approach was shown to qualitatively reproduce the Mott gap [35], we observe that fitting the fragment only (NI procedure) does not predict a gap.

In the case of DET, the transition is clearly visible. We note, however, that calculations performed with a fragment

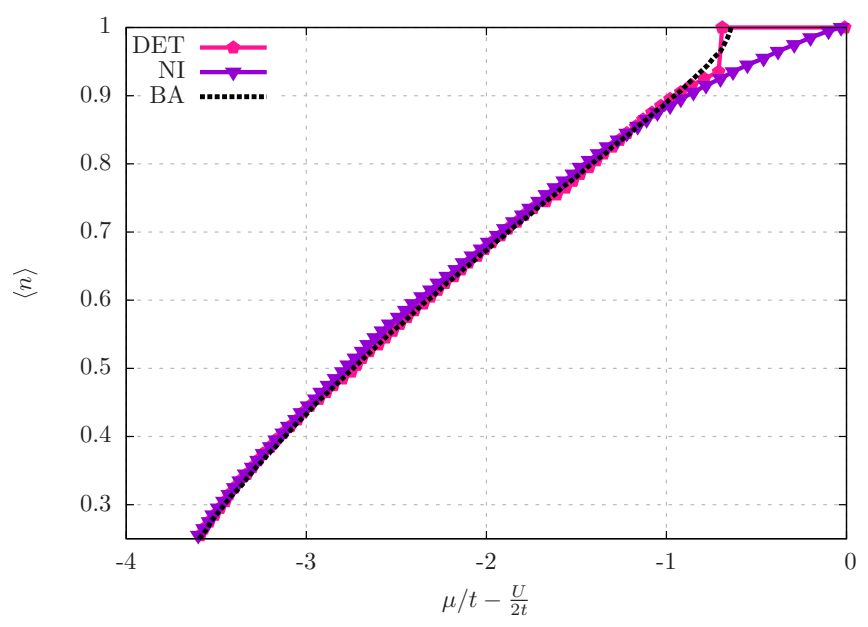

FIG. 5. (Color online) Comparison of lattice filling as a function of chemical potential between the DET embedding scheme with a fragment composed of four sites and the exact Bethe ansatz (BA) results. The data corresponds to $U=4 t$. of two sites predict a somewhat abrupt jump in the $\langle n\rangle(\mu)$ curve around the metal-insulator transition. While this jump seems like an unfortunate consequence of the approximations made in the embedding scheme, the actual position of the Mott transition is rather well reproduced. For $U=4 t$ and $6 t$, the value of the chemical potential is slightly underestimated compared to the Bethe ansatz result. For $U=8 t$, DET(2) coincides very well with the exact answer. In the highly doped regime, the shape of $\langle n\rangle(\mu)$ is also well reproduced. The apparent transitions observed at fillings of around $0.75,0.60$, and 0.45 for $U=4 t, 6 t$, and $8 t$, respectively, correspond to filling fractions where the mean-field calculations no longer break spin symmetry. This unsatisfactory behavior does not however fundamentally change the overall good performance of DET. In Fig. 5, we present an analogous $\langle n\rangle(\mu)$ curve for $U=4 t$ obtained with DET and a fragment size of four. In this case, one observes improvement of the overall results. The value of the chemical potential at which the transition occurs, agrees better with the exact results. Moreover, the description of the highly doped part of the curve is ameliorated. We note, again, that within the NI scheme, even with four sites, the Mott transition is not observed.

\section{Long-range properties}

We now turn our attention to further study two-body correlation functions. As shown in Sec. III B, the expectation value $\left\langle n_{\uparrow} n_{\downarrow}\right\rangle$ is reproduced very well by DET embedding. No significant deviations from exact values are observed. This is not completely surprising as this two-body correlator is local. DMET is therefore particularly well suited to compute such local properties. The question we now wish to address is whether one could access long-range properties from an impurity model. To this end, we study the spin-spin correlation function (SSCF),

$$
\operatorname{SSCF}(j)=\left\langle\hat{S}_{1} \cdot \hat{S}_{j}\right\rangle,
$$

where $\hat{S}_{j}$ is the spin operator at site $j$ [27]. We transform this two-body operator into the embedding basis and evaluate it with the exact solution of the impurity Hamiltonian. We denote the first site of the embedded fragment as site 1 . In Fig. 6, we present the SSCF evaluated for a lattice of eight sites and a fragment of four. Again, we notice that in this case the dimension of the impurity problem is equivalent to the full lattice. The DET scheme is therefore exact. The DMET optimization of the effective potential introduces a discrepancy between the predicted and exact SSCF. Similarly to the energy case, the departure from the exact answer is small but nonnegligible.

Proceeding to systems where the fragment constitutes only a small fraction of the entire system, we compute the SSCF for a lattice of 30 sites with 30,26, and 22 electrons. Results for the value of on-site interaction $U=4 t$ are presented in Fig. 7. The agreement between the embedding schemes and DMRG results is less satisfactory than for other properties. This discrepancy is expected in embedding schemes where the impurity Hamiltonian trades the complexity of the entire lattice by a small fragment connected with bath states. The bath states, whose number is limited by the size of the fragment itself, must account for the rest of the lattice that is not included 


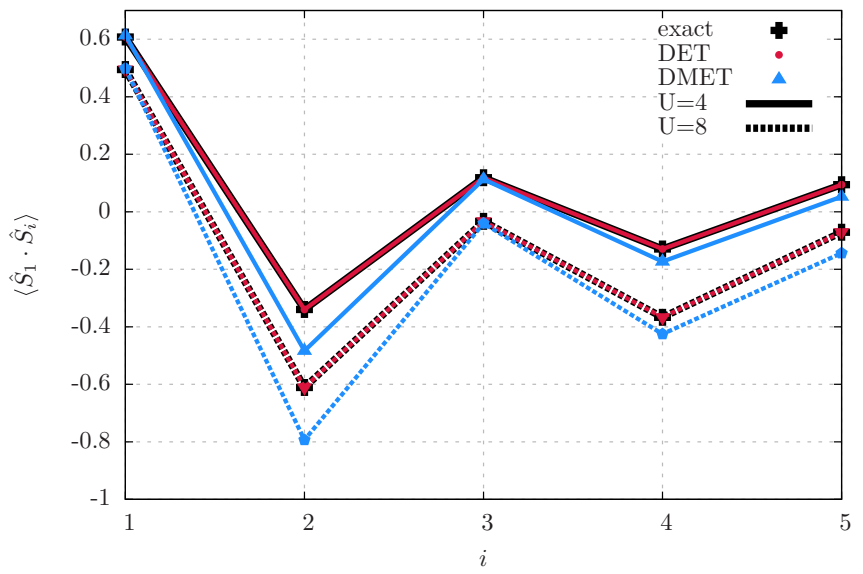

FIG. 6. (Color online) Spin-spin correlation function evaluated for a Hubbard ring of eight sites with DMET and DET embedding. Computations are performed with a fragment of four sites. DMRG results (deemed exact) are provided for comparison. For clarity, the results for $U=8 t$ are shifted down by 0.2 .

in the fragment. In order to illustrate this point more clearly, we plot the partition of the embedding basis single-particle states into distinct sites. For a lattice site $i$, this is defined as

$$
\frac{1}{2} \sum_{d}\left\langle d\left|\hat{P}_{i}\right| d\right\rangle
$$

where the summation runs over embedding states. $\hat{P}_{i}$ is the projector onto site $i$. The factor of one-half accounts for the spin degrees of freedom. Clearly, the sites included in the fragment are completely represented by the impurity Hamiltonian. The contribution to bath states decays rapidly

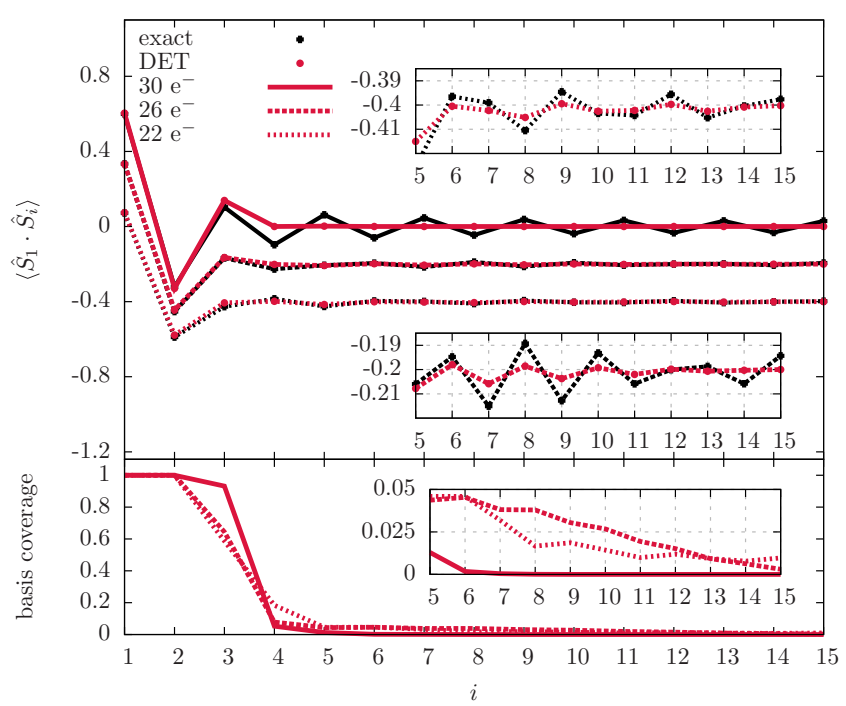

FIG. 7. (Color online) DET and DMET spin-spin correlation functions (SSF) evaluated for a ring of 30 sites with 30, 26, and 22 electrons and $U=4 t$. Calculations are performed with a fragment of two sites. DMRG results (deemed exact) are included for reference. For clarity, data for 26 and 22 electrons are shifted by -0.2 and -0.4 , respectively. The bottom panel presents the basis set coverage (see text for details). with distance from the fragment. This is particularly severe for the half-filled case. In other words, the embedding basis gets screened and the information about spatially distant sites is diminished. This results in a very quick decay of the SSCF. On the other hand, doping the lattice with holes yields a slower spatial decay. As a consequence, the SSCF for doped systems has a nontrivial structure. Indeed, we note that the general trend for this correlator is in agreement with the DMRG reference values, although the absolute values are underestimated. The problem of accounting for long range correlations beyond the size of the embedded fragment is not unique to DMET and also present in DMFT [50].

Let us also note that one may consider evaluating longrange properties by including the inert core states, neglected in the impurity Hamiltonian. While this may improve the description of long-range order (especially when the meanfield is qualitatively correct), we here decided not to include the core states because they are not an explicit part of the correlated calculations.

\section{E. Spin contamination}

In this section, we investigate the expectation value of $\hat{S}^{2}$ over the entire lattice. For the mean-field lattice solution, we evaluate it explicitly with the mean-field wave function. For the impurity Hamiltonian, we project the lattice $\hat{S}^{2}$ operator onto the embedding basis and evaluate it with the impurity density matrices. The data are shown in Table III. Here too, we exclude the contribution from the inert core states.

In the present study, all systems under consideration are singlets. Therefore a nonzero expectation value of the $\hat{S}^{2}$ operator for the impurity Hamiltonian is solely due to spinsymmetry breaking in the Hartree-Fock Hamiltonian, which induces a spin dependence in the embedding basis. The deviation of $\hat{S}^{2}$ from zero is a signature of spin contamination.

The expectation value of $\hat{S}^{2}$ is quite similar for DET and DMET. We also notice a significant decrease in spin contamination - compared to mean-field values-when $\hat{S}^{2}$ is projected onto the embedding basis and evaluated with the impurity wave function. Finally, let us notice that the lowest filling beyond which we could no longer obtain a symmetry broken solution for DMET and DET do not coincide. In particular, for $U=4 t$, spin contamination disappears with DET at a filling of 22 electrons per 30 sites, whereas for DMET,

TABLE III. Expectation values of $\hat{S}^{2}$ for a ring of 30 sites evaluated with the mean-field (MF) and the impurity (imp) wave function. The calculations are performed with a fragment of two sites. See text for discussion.

\begin{tabular}{|c|c|c|c|c|c|c|c|}
\hline & & \multicolumn{6}{|c|}{$\mathrm{NE}$} \\
\hline & & \multicolumn{2}{|c|}{30} & \multicolumn{2}{|c|}{26} & \multicolumn{2}{|c|}{22} \\
\hline & & $\overline{S_{\mathrm{imp}}^{2}}$ & $S_{\mathrm{MF}}^{2}$ & $\overline{S_{\text {imp }}^{2}}$ & $\overline{S_{\mathrm{MF}}^{2}}$ & $S_{\mathrm{imp}}^{2}$ & $S_{\mathrm{MF}}^{2}$ \\
\hline DMET & $(U=4 t)$ & 0.84 & 7.98 & 0.57 & 4.83 & 0.22 & 1.28 \\
\hline DET & $(U=4 t)$ & 0.70 & 9.14 & 0.58 & 5.09 & 0.00 & 0.00 \\
\hline DMET & $(U=8 t)$ & 0.98 & 12.3 & 1.06 & 9.65 & 1.09 & 6.80 \\
\hline DET & $(U=8 t)$ & 0.71 & 13.2 & 1.12 & 10.7 & 1.17 & 7.77 \\
\hline
\end{tabular}


we can still obtain a symmetry broken solution. However, its spin contamination is rather small.

\section{CONCLUSIONS}

We have investigated several DMET variants within a broken spin-symmetry formalism which includes two-body interactions in the lattice mean field. We have shown that the numerical procedure can be simplified and its convergence is greatly improved by only fitting the diagonal of the fragment density matrix. The resulting DET scheme here introduced shows satisfactory accuracy in Hubbard 1D benchmarks. Additionally, we have demonstrated that the DET scheme is exact when the half-filled Hubbard lattice is split into two equal pieces. We deem this property an important guiding principle for defining a robust embedding approximation.

Our numerical DET data for half-filled lattices are in very good agreement with exact results obtained with the Bethe ansatz. This is clear not only for total energies but also for the two-body local correlation function $\left\langle n_{\uparrow} n_{\downarrow}\right\rangle$.

For hole-doped systems, DET yields good Mott gaps and density profiles. Moreover, DET retains full control over the average number of particles, as opposed to the original formulation of Ref. [35]. Both procedures have comparable computational cost except that inclusion of lattice two-body interactions requires an iterative procedure for solving the mean-field Hamiltonian.

As currently formulated, DMET cannot accurately describe long-range correlation functions beyond the fragment size (or, in other words, provide an accurate description of fluctuations beyond the size of the fragment). This is an expected shortcoming inherent to approximations defining an impurity model that is shared by DMFT. We believe that this deficiency constitutes a major challenge in the development of DMET.

Further work to improve and address the many shortcomings discussed in this paper seems warranted. Nonetheless, we believe that DMET is a valuable and promising quantum embedding tool for studying strongly correlated systems, offering high-quality results at very low computational cost.

\section{ACKNOWLEDGMENTS}

I.W.B would like to acknowledge Rayner RodríguezGuzmán for numerous discussions and help with the DMRG calculations. Carlos A. Jiménez-Hoyos is gratefully acknowledged for providing the Bethe ansatz data. This work was supported by the Department of Energy, Office of Basic Energy Sciences, Heavy Element Chemistry program, under Grant No. DE-FG02-04ER15523. G.E.S. is a Welch Foundation Chair (C-0036). J.D. acknowledges support from the Spanish Ministry of Economy and Competitiveness under Grant FIS2012-34479.

\section{APPENDIX: PROPERTIES OF THE SCHMIDT BASIS}

In this appendix, we prove certain properties of the embedding basis obtained via the Schmidt decomposition of a single Slater determinant. Einstein summation convention is used.

\section{Embedding basis}

Let us recall that the embedding basis is obtained from the Schmidt overlap matrix $\mathbb{M}$,

$$
\mathbb{M}_{p q}=\left\langle\phi_{q}\left|\hat{P}_{F}\right| \phi_{p}\right\rangle
$$

( $p$ and $q$ are the hole states), which is diagonalized by a unitary matrix $\mathbb{V}$,

$$
\mathbb{V}^{\dagger} \mathbb{M} \mathbb{V}=d
$$

where $d$ is a diagonal matrix. Let us denote the Hartree-Fock transformation as $\mathbb{D}$. Then the $i$ th fragment and bath states, expressed in terms of the lattice basis take the form

$$
\begin{aligned}
& \mathbb{C}_{\mu i}^{F}=\frac{\mathbb{V}_{p i}^{*} \mathbb{D}_{\mu p}^{F}}{\sqrt{d_{i}}}, \\
& \mathbb{C}_{\mu i}^{B}=\frac{\mathbb{V}_{p i}^{*} \mathbb{D}_{\mu p}^{B}}{\sqrt{1-d_{i}}},
\end{aligned}
$$

where the superscript $F$ and $B$ denote the fragment and the bath states, respectively.

\section{Idempotency of mean-field density matrix in its Schmidt basis}

Let us consider the lattice density matrix $\gamma_{0}$ that has been obtained from the mean-field solution. Projected onto the embedding basis, it will take the following form:

$$
\gamma_{0}=\left(\begin{array}{ll}
\gamma_{0}^{F F} & \gamma_{0}^{F B} \\
\gamma_{0}^{B F} & \gamma_{0}^{B B}
\end{array}\right) .
$$

Using Eq. (A3), for the $F F$ block one obtains

$$
\begin{aligned}
\left(\gamma_{0}^{F F}\right)_{i j} & =\mathbb{C}_{\mu i}^{F *} \mathbb{D}_{\mu r} \mathbb{D}_{v r}^{*} \mathbb{C}_{v j}^{F} \\
& =\mathbb{V}_{p i} \mathbb{D}_{\mu p}^{F *} \mathbb{D}_{\mu r} \mathbb{D}_{v r}^{*} \mathbb{D}_{\nu q}^{F} \mathbb{V}_{q j}^{*} \frac{1}{\sqrt{d_{i} d_{j}}} \\
& =\mathbb{V}_{p i} \mathbb{M}_{r p} \mathbb{M}_{q r} \mathbb{V}_{q j}^{*} \frac{1}{\sqrt{d_{i} d_{j}}}=d_{i} \delta_{i j}
\end{aligned}
$$

In the above, indices $p, q$, and $r$ run over HF hole states, while $\mu$ and $v$ denote the on-site lattice spin orbital.

Analogous straightforward calculations follow for the other blocks. Finally,

$$
\gamma_{0}=\left(\begin{array}{cc}
d & \sqrt{d(1-d)} \\
\sqrt{d(1-d)} & 1-d
\end{array}\right) .
$$

Similarly, one can easily verify that $\gamma_{0}$ is idempotent in the embedding basis and its trace is equal to the dimension of the fragment single-particle basis. On the other hand, the fragment-fragment block of the density matrix need not be idempotent. Indeed, keeping in mind that $0 \leqslant d_{i} \leqslant 1$ (Ref. [38]) and, as we show in Appendix A 4, $\sum_{i} d_{i}$ is the number of electrons per fragment, this situation is highly unlikely. Furthermore, whenever the eigenvalues $d$ are either 0 or 1 , one cannot construct an orthonormal basis according to Eq. (A3).

Additionally, let us stress that the inert core states corresponding to the zero eigenvalues of $\mathbb{M}$ would have vanishing off-diagonal coupling to the fragment states. This is a consequence of the orthogonality of the eigenvectors of $\mathbb{M}$. 


\section{Commutativity of the mean-field density matrix and Fock matrix in the embedding basis}

Let us consider the mean-field Fock matrix in the embedding basis $f=\mathbb{C}^{\dagger} \mathbb{F} \mathbb{C}$, where $\mathbb{F}$ is the lattice Fock matrix. Since $\gamma_{0}$ and $f$ are Hermitian, they commute if and only if their product, $t=f \gamma_{0}$ is Hermitian. Since $t$ and $f$ clearly have the same block structure as $\gamma_{0}$ [Eq. (A4)], one can investigate separately each block of $t$. And so,

$$
\begin{aligned}
t_{i j}^{F F} & =f_{i j}^{F F} d_{j}+f_{i j}^{F B} \sqrt{d_{j}\left(1-d_{j}\right)} \\
& =\sqrt{\frac{d_{j}}{d_{i}}}\left(\mathbb{V}_{p i} \mathbb{D}_{\mu p}^{F *} \mathbb{F}_{\mu \nu} \mathbb{D}_{\nu q} \mathbb{V}_{q j}^{*}\right)=\sqrt{d_{i} d_{j}}\left(\mathbb{V}_{q j}^{*} \epsilon_{q} \mathbb{V}_{q i}\right),
\end{aligned}
$$

where $\epsilon$ is the eigenvalue of $\mathbb{F}$. In the above, one uses the relation that $\mathbb{D}^{F}+\mathbb{D}^{B}=\mathbb{D}$. Similarly,

$$
t_{i j}^{B B}=\sqrt{\left(1-d_{i}\right)\left(1-d_{j}\right)}\left(\mathbb{V}_{q j}^{*} \epsilon_{q} \mathbb{V}_{q i}\right) .
$$

Both of these matrices are manifestly Hermitian. Finally,

$$
t_{i j}^{F B}=\sqrt{\left(1-d_{j}\right) d_{i}}\left(\mathbb{V}_{q j}^{*} \epsilon_{q} \mathbb{V}_{q i}\right)
$$

and

$$
t_{i j}^{B F}=\sqrt{\left(1-d_{i}\right) d_{j}}\left(\mathbb{V}_{q j}^{*} \epsilon_{q} \mathbb{V}_{q i}\right) .
$$

Clearly $t_{i j}^{* F B}=t_{j i}^{B F}$, hence $t$ is Hermitian and $\gamma_{0}$ and $f$ commute.

\section{Fragment states can be chosen as bare fermion states}

Let us denote the number of bare fermion single-particle states as $M$ and the number of fragment states as $N$. The fragment basis can be now expressed as

$$
\tilde{\mathbb{C}}^{F}=\left(\begin{array}{c}
\mathbb{C}^{F} \\
0
\end{array}\right),
$$

which is a $M \times N$ matrix with $\mathbb{C}_{F}$ being a $N \times N$ matrix. Since $\mathbb{C}^{F}$ is a linear transformation that preserves vectors length, it is unitary,

$$
\mathbb{C}^{F^{\dagger}} \mathbb{C}=\mathbb{C}^{F^{\dagger}}=\mathbb{I}_{N \times N} .
$$

The full embedding basis takes the form

$$
\mathbb{C}^{I}=\left(\begin{array}{cc}
\mathbb{C}^{F} & 0 \\
0 & \mathbb{C}^{B}
\end{array}\right),
$$

which satisfies $\mathbb{C}^{I \dagger} \mathbb{C}^{I}=\mathbb{I}_{2 N \times 2 N}$, but $\mathbb{C} \mathbb{C}^{I \dagger} \neq \mathbb{I}_{M \times M}$ unless, of course $N=M / 2$. We can now transform the embedding basis with a unitary transformation

$$
\mathbb{U}=\left(\begin{array}{cc}
\mathbb{C}^{F \dagger} & 0 \\
0 & \mathbb{I}
\end{array}\right),
$$

such that

$$
\mathbb{C}^{I \prime}=\mathbb{U} \mathbb{C}^{I}
$$

is expressed in the fragment bare fermion basis. We note that such unitary transformation does not affect the idempotency of $\gamma_{0}$ and its commutativity with $f$.

Finally, let us note that this transformation does not affect the trace of $\gamma_{0}$ taken over the $F F$ block. Since the fragment basis is now equivalent to the bare fermion basis, we see that the trace of $\gamma_{0}^{F}$ (hence $\sum_{i} d_{i}$ ) must be equal to the number of electrons per fragment, provided that the mean-field solution does not break translational symmetry (again, assuming that one works with an extended unit cell chosen as fragment).
[1] G. K.-L. Chan and S. Sharma, Annu. Rev. Phys. Chem. 62, 465 (2011).

[2] E. Morosan, D. Natelson, A. H. Nevidomskyy, and Q. Si, Adv. Mater. 24, 4896 (2012).

[3] E. Dagotto, Rev. Mod. Phys. 66, 763 (1994).

[4] J. Bednorz and K. Mller, Z. Phys. B Con. Mat. 64, 189 (1986).

[5] E. Dagotto, Science 309, 257 (2005).

[6] A. Moreo, S. Yunoki, and E. Dagotto, Science 283, 2034 (1999).

[7] C. Gros, R. Joynt, and T. M. Rice, Phys. Rev. B 36, 381 (1987).

[8] S. Sorella, Phys. Rev. B 71, 241103 (2005).

[9] E. Neuscamman, C. J. Umrigar, and G. K.-L. Chan, Phys. Rev. B 85, 045103 (2012).

[10] S. Zhang, J. Carlson, and J. E. Gubernatis, Phys. Rev. Lett. 78, 4486 (1997).

[11] C.-C. Chang and S. Zhang, Phys. Rev. Lett. 104, 116402 (2010).

[12] T. Yanagisawa, S. Koike, and K. Yamaji, Phys. Rev. B 64, 184509 (2001).

[13] W. Metzner and D. Vollhardt, Phys. Rev. Lett. 62, 324 (1989).

[14] M. Jarrell, Phys. Rev. Lett. 69, 168 (1992).

[15] M. J. Rozenberg, X. Y. Zhang, and G. Kotliar, Phys. Rev. Lett. 69, 1236 (1992).

[16] A. Georges and W. Krauth, Phys. Rev. Lett. 69, 1240 (1992).

[17] M. Caffarel and W. Krauth, Phys. Rev. Lett. 72, 1545 (1994).
[18] G. Sordi, K. Haule, and A.-M. S. Tremblay, Phys. Rev. Lett. 104, 226402 (2010).

[19] E. Koch, G. Sangiovanni, and O. Gunnarsson, Phys. Rev. B 78, 115102 (2008).

[20] M. Balzer, B. Kyung, D. Snchal, A.-M. S. Tremblay, and M. Potthoff, EPL 85, 17002 (2009).

[21] M. Capone, M. Civelli, S. S. Kancharla, C. Castellani, and G. Kotliar, Phys. Rev. B 69, 195105 (2004).

[22] A. Georges, G. Kotliar, W. Krauth, and M. J. Rozenberg, Rev. Mod. Phys. 68, 13 (1996).

[23] S. R. White, Phys. Rev. Lett. 69, 2863 (1992).

[24] J. Dukelsky and S. Pittel, Rep. Prog. Phys. 67, 513 (2004).

[25] U. Schollwöck, Rev. Mod. Phys. 77, 259 (2005).

[26] T. Xiang, Phys. Rev. B 53, R10445 (1996).

[27] R. Rodríguez-Guzmán, C. A. Jiménez-Hoyos, R. Schutski, and G. E. Scuseria, Phys. Rev. B 87, 235129 (2013).

[28] G. E. Scuseria, C. A. Jimenez-Hoyos, T. M. Henderson, K. Samanta, and J. K. Ellis, J. Chem. Phys. 135, 124108 (2011).

[29] C. A. Jimenez-Hoyos, T. M. Henderson, T. Tsuchimochi, and G. E. Scuseria, J. Chem. Phys. 136, 164109 (2012).

[30] R. Rodríguez-Guzmán, K. W. Schmid, C. A. Jiménez-Hoyos, and G. E. Scuseria, Phys. Rev. B 85, 245130 (2012).

[31] O. Juillet and R. Frésard, Phys. Rev. B 87, 115136 (2013). 
[32] N. Tomita, Phys. Rev. B 69, 045110 (2004).

[33] D. Baeriswyl, D. Eichenberger, and M. Menteshashvili, New. J. Phys. 11, 075010 (2009).

[34] N. Lanatà, H. U. R. Strand, X. Dai, and B. Hellsing, Phys. Rev. B 85, 035133 (2012).

[35] G. Knizia and G. K.-L. Chan, Phys. Rev. Lett. 109, 186404 (2012).

[36] G. Knizia and G. K.-L. Chan, J. Chem. Theory Comput. 9, 1428 (2013).

[37] G. H. Booth and G. K.-L. Chan, arXiv:1309.2320.

[38] I. Klich, J. Phys. A: Math. Gen. 39, L85 (2006).

[39] Symmetry breaking solutions were presented for the 2D Hubbard model in Ref. [35], however, a systematic assessment of spin symmetry breaking was not included .

[40] I. Peschel, Braz. J. Phys. 42, 267 (2012).

[41] P. Ring and P. Schuck, The Nuclear Many-Body Problem (Springer, Berlin, 2004).

[42] We do not discuss the possibility of breaking the Hamiltonian symmetry, particularly the $\hat{S}^{2}$ symmetry by a spin-dependent effective potential introduced in Eq. (3). This is a possible route for further developments of the model.
[43] E. H. Lieb and F. Y. Wu, Phys. Rev. Lett. 20, 1445 (1968).

[44] V. L. Campo and K. Capelle, Phys. Rev. A 72, 061602 (2005).

[45] A. Snyder, I. Tanabe, and T. De Silva, Phys. Rev. A 83, 063632 (2011).

[46] N. A. Lima, M. F. Silva, L. N. Oliveira, and K. Capelle, Phys. Rev. Lett. 90, 146402 (2003).

[47] M. Balzer, W. Hanke, and M. Potthoff, Phys. Rev. B 77, 045133 (2008).

[48] A. Go and G. S. Jeon, J. Phys.: Condens. Matter 21, 485602 (2009).

[49] To the best of our understanding, the chemical potential for a given density in Ref. [35] was extracted from the mean-field Fock matrix. In the present work, we do not use mean-field quantities apart from the embedding basis itself. In Figs. 4 and 5, we do not include results with $\mathrm{NI}_{F}$ as we do not deem them directly comparable to our results. We refer the reader to Ref. [35] for a discussion of the $\mathrm{NI}_{F}$ results.

[50] A. Toschi, A. A. Katanin, and K. Held, Phys. Rev. B 75, 045118 (2007). 